\title{
Modulation of the Mitogenic Response of Lymphocytes from Young and Aged Individuals by Prostaglandins and Indomethacin
}

\author{
K. Murali Krishna Rao, ${ }^{*}$ Stanley A. Schwartz,* and Robert A. Good \\ Memorial Sloan-Kettering Cancer Center, New York, New York 10021, and Department of Pediatrics, * \\ University of Michigan, Ann Arbor, Michigan 48109
}

Received November 3, 1978

\begin{abstract}
The effect of indomethacin (IND) on the mitogenic responses of human peripheral blood lymphocytes was dependent upon the dose of mitogen employed; in contrast, the effect of prostaglandins (PGs) was independent of the mitogen dose. When a single concentration of mitogen was used to study the effect of IND on blastogenesis, extremely variable results were observed, even among a healthy population group. This variability within the group was reduced to a minimum when mitogen dose-response studies were performed with and without IND and maximal blastogenic responses were compared. Employing such a criterion, no age-related differences were noted on addition of IND. Kinetic experiments revealed that significant quantities of PGs must be present very early during the culture period to influence the lectin-induced mitogenesis. These findings, together with recently published data from other laboratories, lead us to conclude that endogenously synthesized PGs may not be able to influence the lectin-induced mitogenic process. The effect of IND observed at submitogenic doses of lectins may be due to other pharmacological actions such as its effect on cyclic AMP-dependent protein kinases.
\end{abstract}

\section{INTRODUCTION}

Regulation of immune function by hormones, vasoactive amines, and antiinflammatory drugs is presently in the forefront of immunological research. Receptors for histamine, the $\beta$-mimetic catecholamines, and the prostaglandins (PGs) of the E and A Series (for review, see 1) have been demonstrated on human and murine lymphocytes. Moreover, PGs have been shown to be active in the inhibition of T-cell functions (2), control of the $19 \mathrm{~S}$ antibody response to sheep erythrocytes (3), inhibition of cytolytic activity (4), and inhibition of lymphokine secretion from sensitized human lymphocytes (5). Thus, PGs are being attributed a prominent role as modulators of immune function in a variety of conditions. The evidence implicating PGs in immune regulation is based on the ability of a prostaglandin synthetase inhibitor, indomethacin (IND), to enhance the mitogenic responses of lectin-stimulated peripheral blood lymphocytes (PBL) in vitro and the ability of exogenously added PGs to inhibit lectin-induced mitogenic responses. PG secretion by peripheral blood mononuclear cells has been attributed to a glass adherent cell population (6), and increased production of PGs by these cells has been implicated in the depressed cellular immunity manifested in Hodgkin's disease 
(7), and aging (8). However, the data on the effect of IND on phytohemagglutinin (PHA) and concanavalin A (Con A)-stimulated mitogenesis is conflicting and inconsistent. Novogrodsky et al. (9) reported that IND failed to show any enhancing effect in Con A- and PHA-stimulated cultures. In all these studies the experiments were performed using a single mitogen dose.

In this paper we demonstrate that the effect of IND $(1 \mu \mathrm{g} / \mathrm{ml})$ on the mitogenic response of PBL is dependent upon the concentration of the mitogen used for lymphocyte stimulation. Further, since the concentration of mitogen for maximal blastogenic response was found to vary from individual to individual, dose-response curves to several mitogens, with and without IND, or exogenous PG were determined on PBL from healthy young and aged volunteer donors. We report here the results of these experiments, which suggest that the apparent enhancement of lectin-induced lymphocyte mitogenesis by IND may involve pharmacological actions other than inhibition of prostaglandin synthetase.

\section{MATERIALS AND METHODS}

Donors. Blood from young and aged healthy volunteer donors was freshly obtained from a peripheral vein. All donors gave informed consent consistent with institutional and Department of Health, Education, and Welfare policies. The aging population ranged from 65 to 74 years with a mean age of 69.3 years; the young adult population ranged from 23 to 48 years with a mean of 32.8 years.

Isolation of lymphocytes. Peripheral blood mononuclear leukocytes were prepared from fresh heparinized blood $(20 \mathrm{U} / \mathrm{ml})$ by a modification of the density gradient technique of Böyum (10). The mononuclear cell band was harvested and washed three times with $\mathrm{CA}^{2+}$ - and $\mathrm{MG}^{2+}$-free Hanks' balanced salt solution (Grand Island Biological Co.) and resuspended in RPMI 1640 medium (Grand Island Biological Co.) containing 10\% heat-inactivated fetal calf serum (Associated Biomedic Systems, Inc., Buffalo, N.Y.), $80 \mu \mathrm{g}$ gentamicin/ml (Schering Corp., Kenilworth, N.J.), and $300 \mu \mathrm{g}$ of fresh glutamine $/ \mathrm{ml}$.

Drugs. $\mathrm{PGE}_{1}, \mathrm{PGE}_{2}$, and IND were obtained from a commercial supplier (Sigma Chemical Co., St. Louis, Mo.). $\mathrm{PGE}_{1}$ and $\mathrm{PGE}_{2}$ were dissolved to a concentration of $1 \mathrm{mg} / \mathrm{ml}$, and IND to $10 \mathrm{mg} / \mathrm{ml}$ in $95 \%$ ethyl alcohol. Further dilutions were made in RPMI 1640 medium, aliquoted and stored frozen at $-20^{\circ} \mathrm{C}$. Ethanol alone, at the final concentration obtained after adding the drugs to the cultures $(\geq 0.01 \%)$ had no effect on the mitogenic response in the majority of the subjects. However, an occasional individual showed a slight enhancement with the ethanol alone.

Cell cultures. Cells were cultured in round-bottom microtiter plates (Falcon Plastics, Oxnard, Calif.) at a concentration of $5 \times 10^{4}$ cells in $200 \mu \mathrm{l}$. In some experiments $2 \times 10^{5}$ cells were cultured in $200 \mu \mathrm{l}$ of $20 \%$ FCS, where noted. Mitogens and drugs were added directly to the wells. The final volume of all the cultures was adjusted to $240 \mu \mathrm{l}$ and the cells were incubated for $72 \mathrm{hr}$ at $37^{\circ} \mathrm{C}$ in an atmosphere consisting of $5 \% \mathrm{CO}_{2}$ and $95 \%$ air. At the end of $72 \mathrm{hr}, 2 \mu \mathrm{Ci}$ of [methyl $\left.-{ }^{3} \mathrm{H}\right]$ thymidine, specific activity $6.7 \mathrm{Ci} / \mathrm{mmol}\left(\left[{ }^{3} \mathrm{H}\right]\right.$ thymidine, New England Nuclear, Boston, Mass.) was added to each well and cultured for an additional 18 hr. The cells were harvested by an automatic cell harvester (Flow Laboratories, Rockville, Md.) and incorporation of $\left[{ }^{3} \mathrm{H}\right]$ thymidine was measured by liquid scintillation spectroscopy. Dose-response curves to PHA-P (Difco Laboratories, 
Detroit, Mich.) and Con A (Pharmacia Fine Chemicals, Piscataway, N.J.) were determined with and without the addition of the various pharmacologic agents. All cultures were performed in triplicate and the results expressed as mean \pm 1 standard deviation.

The percentage change induced by IND was calculated according to the formula $\left(C_{1}-C\right) / C \times 100$, where $C_{\mathrm{I}}$ is counts per minute in the presence of mitogen and IND, and $C$ is counts per minute in the presence of mitogen only.

\section{RESULTS}

\section{Effect of IND on Dose-Response Curves to Con A and PHA}

Figure 1 shows two representative dose-response curves to Con $\mathrm{A}$ with and without IND, using $5 \times 10^{4}$ cells per well. In Fig. 1a, IND evoked a change in sensitivity of the lymphocytes to mitogenesis by Con $A$, resulting in a broadened maximum, with comparable responses at both 5 and $10 \mu \mathrm{g} / \mathrm{ml}$ of Con $A$. Had a single concentration of $5 \mu \mathrm{g} / \mathrm{ml}$ of Con A been solely selected for comparing the effects of IND, it would appear that IND caused a marked enhancement of mitogenesis. However, when maximal mitogenic responses are compared with and without IND,
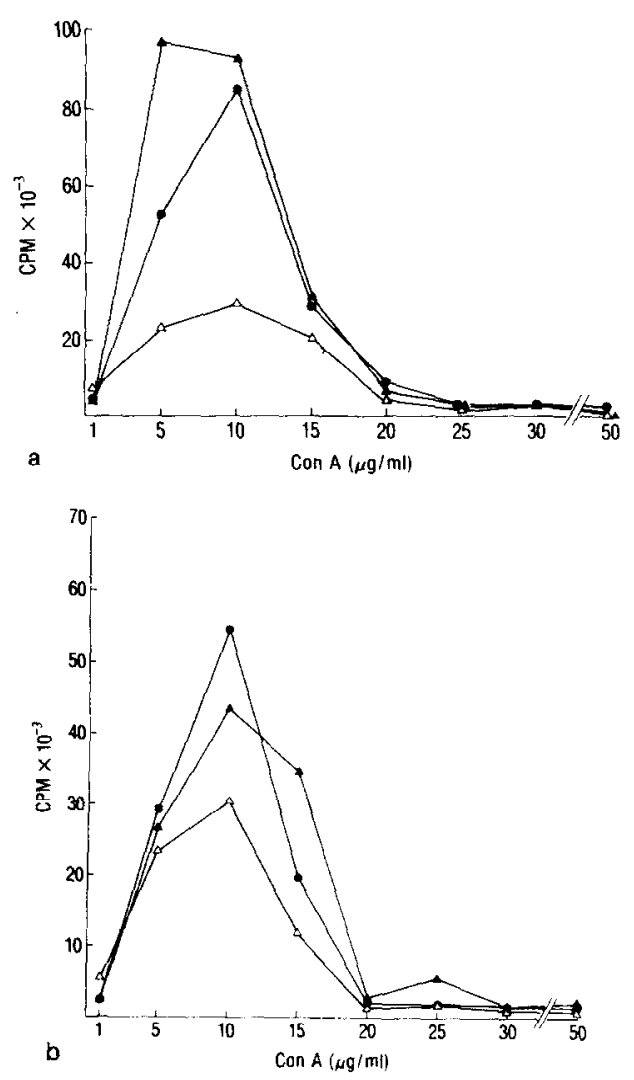

FIG. 1. Effect of IND $(1 \mu \mathrm{g} / \mathrm{ml})$ and PGE $_{2}\left(4.7 \times 10^{-8} M\right)$ on the Con A-induced mitogenic response of PBL. Representative dose-response curves from two donors (a and b). $\bullet$, Con A; $\Delta$, Con A + IND; $\triangle$, Con $\mathrm{A}+\mathrm{PGE}_{2}$. 
it can be seen that IND had no significant effect on the maximal response. In Fig. 1b, the Con A dose for optimum response remains unaltered in the presence or absence of IND, but the magnitude of the blastogenic response at 10 and $15 \mu \mathrm{g} / \mathrm{ml}$ of the mitogen differs significantly on adding IND. These two dose-response curves illustrate the great variability of the effect of IND. Further, among the persons tested in this study, the optimum Con A dose varied from 5 to $25 \mu \mathrm{g} / \mathrm{ml}$ under similar conditions. For this reason, we propose that a comparison of the maximal mitogenic response in the presence and absence of IND is a preferable measure of the immunoregulatory effect of this agent on lectin-induced mitogenesis.

Figure 2 shows dose-response curves to PHA-P with and without IND. In Fig. $2 a$, IND does not exert an effect on mitogenesis at any lectin dose tested. With PBL from another healthy donor there was a shift in the optimum to a higher mitogen dose in the presence of IND but no change in the magnitude of the maximal mitogenic response (Fig. 2b), thus indicating that the use of a full dose-response curve is a more informative parameter of the effect of IND on any lectin-induced mitogenesis.

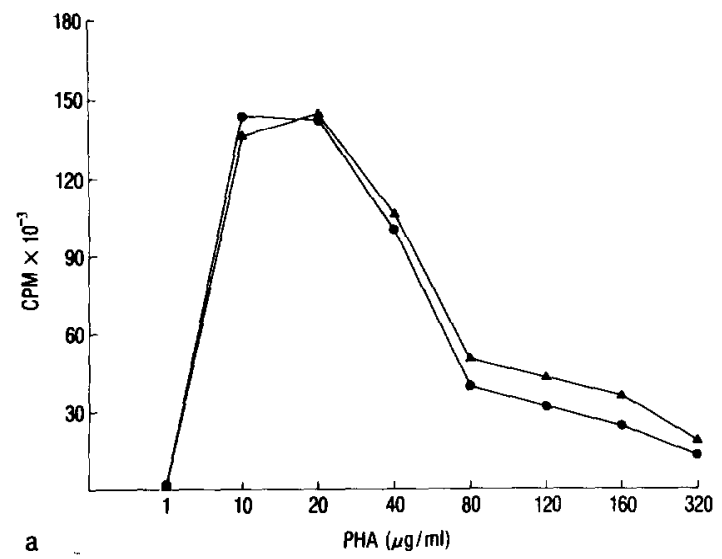

a

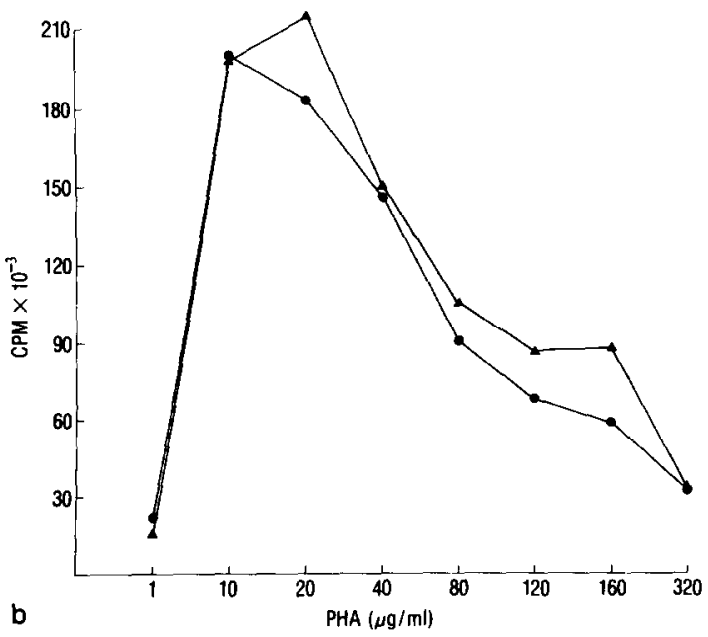

FIG. 2. Effect of IND $(1 \mu \mathrm{g} / \mathrm{ml})$ on the mitogenic response of PBL induced by PHA. Representative dose-response curves from two donors (a and b). 9, PHA; $\Delta$, PHA + IND. 


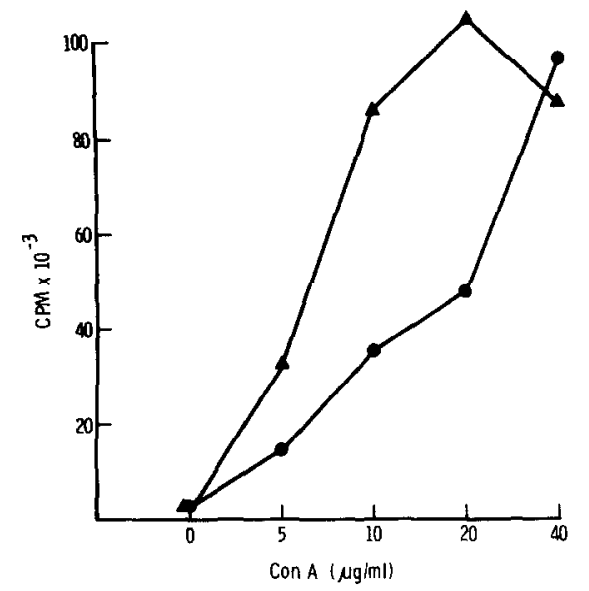

FiG. 3. Effect of IND $(1 \mu \mathrm{g} / \mathrm{ml})$ on the dose-response curve of Con A using $2 \times 10^{5}$ cells per well and $20 \%$ FCS. $\bullet$, Con A; $A$, Con A + IND.

The optimum mitogen concentration for maximal blastogenic response varies considerably with the experimental conditions employed. When experiments were performed using $2 \times 10^{5}$ cells per well and 20\% FCS the results shown in Fig. 3 were obtained. It can be seen that the mitogenic response does not show a downtrend even at $40 \mu \mathrm{g} / \mathrm{ml}$ of Con A; further, when IND is present in the culture the optimum shifts to $20 \mu \mathrm{g} / \mathrm{ml}$ of Con A and the mitogenic response begins to decrease at 40 $\mu \mathrm{g} / \mathrm{ml}$, again demonstrating that at mitogen concentrations approaching optimal, IND fails to cause enhancement in blastogenesis. However, under these conditions, if the change induced by IND is expressed as the percentage change at a single mitogen concentration (e.g., $5 \mu \mathrm{g} / \mathrm{ml}$ Con A) the data would appear as shown in Fig. 4. We would like to point out that $5 \mu \mathrm{g} / \mathrm{ml}$ Con A under these conditions was a suboptimal dose. Further, the data as presented in Fig. 4 reveal that some normal individuals show as much as $337 \%$ enhancement in the presence of IND.

\section{Effect of IND on Lymphocytes from Young and Aged Individuals}

Table 1 summarizes the results of the IND effect on Con A-induced mitogenesis at a cell concentration of 50,000 PBL per well. The effect of IND was determined by performing dose-response curves to Con $A$ with and without IND and comparing the maximal mitogenic responses. In 8 of 11 healthy young persons, IND failed to show a singificant change $(P$ value $<0.05)$ in the maximal blastogenic response; with PBL from one individual there was enhancement of the peak response with IND, whereas PBL from another donor yielded a lower maximal mitogenic response in the presence of IND. Using PBL from 10 healthy aged individuals, addition of IND to mitogen-stimulated cultures caused no change in 7 , enhancement in 2, and suppression in 1, thus indicating no difference in response to IND between the two groups.

\section{Effect of PGE $E_{2}$ on the Mitogenic Response}

Among the 16 persons, both young and aged, studied for PG influence, there was consistent suppression of transformation responses to all doses of Con A tested at a 


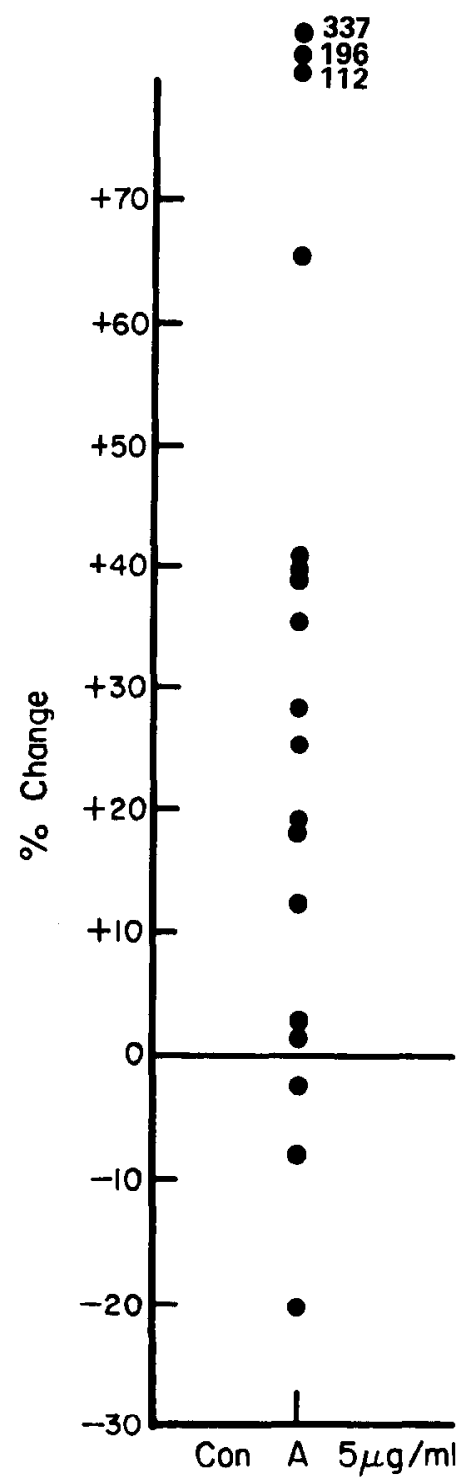

Fig. 4. Effect of IND $(1 \mu \mathrm{g} / \mathrm{ml})$ on Con A-stimulated mitogenic responses expressed as a percentage change, using $2 \times 10^{5}$ cells per well and $20 \%$ FCS.

final concentration of $2.35 \times 10^{-8} M \mathrm{PGE}_{2}$. The only exception was a 23-year-old female whose PBL showed a slight enhancement of mitogenesis when $\mathrm{PGE}_{2}$ was added. A typical dose-response to Con $A$ at various concentrations of $\mathrm{PGE}_{2}$ ranging from $2.35 \times 10^{-8} M$ to $1.8 \times 10^{-7} M$ is shown in Fig. 5 .

Time-Course Experiments with $P G E_{2}$

$\mathrm{PGE}_{2}$ was added to the wells at various time intervals after initiation of the cultures with Con A. Figure 6 shows that the addition of $\mathrm{PGE}_{2}$ at 0 and $2 \mathrm{hr}$ suppressed the mitogenic response, but by $4 \mathrm{hr}$ the suppression was partially lost and addition of $\mathrm{PGE}_{2}$ at $16 \mathrm{hr}$ failed to suppress mitogenesis. 
TABLE 1

Effect of Indomethacin on the Mitogenic Response of PBL to Con A

\begin{tabular}{|c|c|c|c|c|c|}
\hline \multirow[b]{2}{*}{ Donor No. } & \multirow[b]{2}{*}{ Age } & \multirow[b]{2}{*}{ Sex } & \multicolumn{3}{|c|}{$\left[{ }^{3} \mathrm{H}\right]$ Thymidine incorporation $(\mathrm{cpm})$ in response to mitogens ${ }^{a}$} \\
\hline & & & Con A & Con $A+I N D$ & Change $^{b}$ \\
\hline \multicolumn{6}{|c|}{ Young individuals } \\
\hline 1 & 48 & M & $67,371 \pm 16,268$ & $56,885 \pm 1,458$ & 0 \\
\hline 2 & 31 & $\mathbf{M}$ & $33,972 \pm 11,370$ & $27,261 \pm 13,264$ & 0 \\
\hline 3 & 25 & $\mathrm{~F}$ & $77,734 \pm 10,430$ & $31,428 \pm 5,497$ & - \\
\hline 4 & 47 & $\mathbf{M}$ & $35,358 \pm 4,186$ & $27,983 \pm 3,071$ & 0 \\
\hline 5 & 37 & $\mathbf{F}$ & $67,721 \pm 5,988$ & $67,476 \pm 12,055$ & 0 \\
\hline 6 & 23 & $\mathbf{F}$ & $126,827 \pm 17,962$ & $129,646 \pm 21,034$ & 0 \\
\hline 7 & 37 & $\mathbf{M}$ & $84,264 \pm 5,238$ & $94,409 \pm 9,424$ & 0 \\
\hline 8 & 31 & $\mathbf{M}$ & $49,815 \pm 9,098$ & $39,812 \pm 5,414$ & 0 \\
\hline 9 & 23 & $\mathbf{F}$ & $75,206 \pm 6,222$ & $107,949 \pm 11,391$ & + \\
\hline 10 & 24 & $\mathbf{M}$ & $52,830 \pm 6,647$ & $50,845 \pm 7,452$ & 0 \\
\hline 11 & 35 & $\mathbf{M}$ & $129,637 \pm 4,012$ & $118,716 \pm 5,091$ & 0 \\
\hline \multicolumn{6}{|c|}{ Aged individuals } \\
\hline 12 & 69 & $\mathrm{~F}$ & $106,628 \pm 12,510$ & $47,442 \pm 7,970$ & - \\
\hline 13 & 74 & $F$ & $49,625 \pm 18,467$ & $112,559 \pm 11,124$ & + \\
\hline 14 & 72 & $\mathbf{M}$ & $26,761 \pm 4,122$ & $27,779 \pm 2,793$ & 0 \\
\hline 15 & 65 & $\mathbf{F}$ & $71,033 \pm 7,782$ & $73,242 \pm 4,658$ & 0 \\
\hline 16 & 67 & $\mathrm{~F}$ & $32,036 \pm 4,770$ & $35,273 \pm 3,734$ & 0 \\
\hline 17 & 70 & $\mathbf{F}$ & $17,538 \pm 2,181$ & $26,523 \pm 5,378$ & 0 \\
\hline 18 & 71 & $\mathbf{M}$ & $14,282 \pm 3,280$ & $33,048 \pm 3,667$ & + \\
\hline 19 & 67 & $F$ & $18,197 \pm 2,865$ & $25,372 \pm 4,894$ & 0 \\
\hline 20 & 69 & $\mathbf{M}$ & $8,356 \pm 908$ & $9,570 \pm \quad 744$ & 0 \\
\hline 21 & 69 & $\mathbf{M}$ & $88,228 \pm 7,336$ & $89,788 \pm 10,100$ & 0 \\
\hline
\end{tabular}

" $5 \times 10^{4}$ donor lymphocytes were cultured for $72 \mathrm{hr}$ in the presence of various Con A doses with and without added IND $(1 \mu \mathrm{g} / \mathrm{ml})$ and labeled with $2 \mu \mathrm{Ci}\left[{ }^{3} \mathrm{H}\right]$ thymidine as described under $\mathrm{Ma}$ terials and Methods. The maximal responses $\pm 1 \mathrm{SD}$ are presented for each dose-response curve.

${ }^{b} 0$, No change; +, enhancement; -, suppression; significant at $P$ value $<0.05$.

\section{DISCUSSION}

The hypothesis that PGs may have a profound influence on the immune response and that abnormalities of PG metabolism may explain some immune-deficient states is an exciting proposition. The influence of PGs on the immune response is based, in part, on the effects of IND on lectin-induced in vitro mitogenic responses and partly on the effects of the addition of exogenous PGs to cultures of PBL. However, the data on the effect of IND on the in vitro lectin-induced mitogenic response of PBL are conflicting and inconsistent. It was originally reported that IND had an enhancing effect, at all concentrations of the mitogen tested (6). Later reports indicated that the effect of IND is dependent upon the mitogen dose (11). Other investigators observed that IND failed to cause any change in the mitogenic response induced by Con A and PHA (9). Our results demonstrate that the effect of IND depends upon the dose of the mitogen employed. Further, at optimal concentrations of the mitogen, IND fails to cause any enhancement of blastogenesis, which may explain why some investigators were unable to observe enhancement of mitogenesis by IND in their studies. 


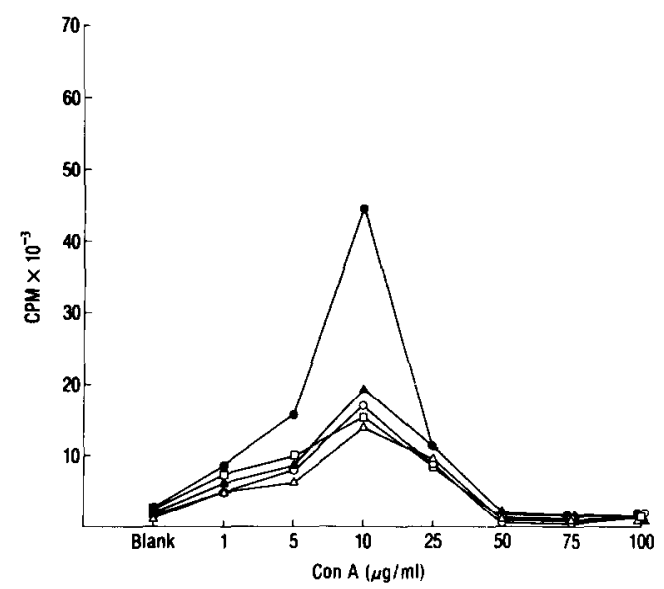

FIG. 5. Effect of $\mathrm{PGE}_{2}$ on the mitogenic response of Con A-stimulated PBL. - Con A; $\triangle$, Con $\mathrm{A}+\mathrm{PGE}_{2}\left(2.35 \times 10^{-8} M\right) ; \triangle$, Con $\mathrm{A}+\mathrm{PGE}_{2}\left(4.7 \times 10^{-8} M\right) ; \mathrm{O}, \mathrm{Con} \mathrm{A}+\mathrm{PGE}_{2}\left(9.4 \times 10^{-8} M\right) ; \square$, Con $\mathrm{A}+\mathrm{PGE}_{2}\left(1.8 \times 10^{-7} M\right)$.

This observation of dose dependency of the IND effect raises an important issue. Our own experiments and other studies (12) indicate that no single mitogen dose is optimal for all individuals. Therefore, the employment of a single dose of mitogen could lead to optimal responses by PBL from some donors and suboptimal responses by others. This variation in dose response may become more critical when comparing a normal population with a disease population. In Fig. 4 we report the data using a single concentration of Con A $(5 \mu \mathrm{g} / \mathrm{ml})$ expressing the results as a percentage change. We wish to point out the great variability of the effect of IND as presented in that figure; however, these individual differences disappear when dose-response studies are performed and the optimal mitogenic responses are compared with and without IND. (Table 1). It should also be noted that in Fig. 4 three healthy individuals showed as much enhancement at a concentration of 5 $\mu \mathrm{g} / \mathrm{ml}$ of Con A as had been previously reported for Hodgkin's disease patients (7). Based on these observations, we conclude that the use of a single mitogen dose for comparing the effect of IND on different population groups could lead to quite variable results, and may further yield misleading conclusions, especially, when the

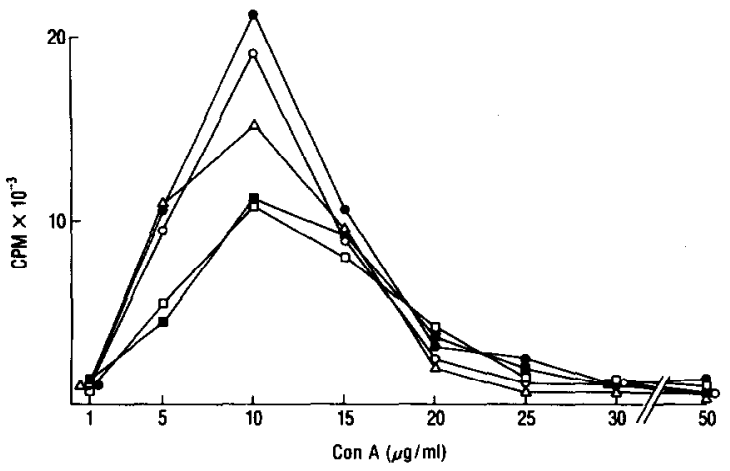

FIG. 6. Effect of the addition of $\mathrm{PGE}_{2}\left(4.7 \times 10^{-8} \mathrm{M}\right)$ at various time intervals after the initiation of the culture of PBL with Con A. $\bullet$, Con A; $\square$, Con A + $\mathrm{PGE}_{2}(0 \mathrm{hr}) ; \mathbf{\square}$, Con A + $\mathrm{PGE}_{2}(2 \mathrm{hr}) ; \triangle$, Con $\mathrm{A}+\mathrm{PGE}_{2}(4 \mathrm{hr}) ; \mathrm{O}, \mathrm{Con} \mathrm{A}+\mathrm{PGE}_{2}(16 \mathrm{hr})$. 
sample size is small. However, when dose-response curves are performed with and without IND and the maximal mitogenic responses are compared, the variation within a healthy control population is minimal. Therefore, we propose that it is essential to perform such dose-response experiments with and without IND to draw meaningful conclusions between any two population groups.

In the above discussion we endeavored to explain the possible reasons for discrepancies in the literature pertaining to the effect of IND on lectin-induced lymphocyte transformation. Now, we shall address ourselves to the more fundamental issue of whether the effect of IND is due to its actions on the inhibition of prostaglandin synthesis or due to some other pharmacological activity of IND. Second, is the effect of PG physiological or only pharmacological?

Figure 1 reveals that the effect of IND was dependent upon the dose of the mitogen; in contrast, the effect of $\mathrm{PGE}_{2}$ was independent of mitogen concentration. That is, the biological effect of IND was not diametrically opposite to that of PG. If IND were exerting its effect by inhibiting PG synthesis one might expect enhancement of the transformation response even at the optimum concentration of the mitogen.

Next let us consider the effect of PG itself. If endogenously synthesized PGs suppress the lectin-induced mitogenic process as predicted by the model, it should follow that the addition of IND might likewise enhance the mitogenic process at any concentration of the mitogen. The inability of IND to show enhancement of blastogenesis at optimal doses of the mitogen is explained on the basis of a lack of sensitivity to $P G$ as the mitogen concentration is increased (11). However, in Fig. 1 it can be seen that exogenous PG was still capable of suppressing the mitogenic response at the optimal dose of Con A. This observation does not support the concept of a loss of sensitivity to PG at higher mitogen concentration.

Figure 5 shows that there was consistent suppression of transformation responses at the optimal dose of Con $\mathrm{A}$ with concentrations of $\mathrm{PGE}_{2}$ ranging from $2.35 \times 10^{-8}$ to $1.8 \times 10^{-7} M$. It should be noted that raising the concentration of $P G$ eightfold did not cause more suppression than at $2.35 \times 10^{-8} \mathrm{M}$ which is considered a physiological concentration. This experiment indicates that if endogenously produced PGs were already exerting a significant suppression in the culture, then addition of a small amount of exogenous PG would not be expected to cause a noticeable change. The fact that the mitogenic response is so exquisitely sensitive to exogenous PGs leads one to consider the role of endogenously produced PG more critically.

In the studies implicating the role of endogenous PGs in pathological states, the levels of PG produced by in vitro cultures of PBL were measured at the end of a 48-hr culture period. Kinetic studies on PG synthesis by PBL, in vitro, have shown that it takes approximately $16 \mathrm{hr}$ or more following a mitogenic stimulus to observe a noticeable change in the production of PG in the culture as compared to nonstimulated cells $(11,13)$. However, within minutes after the addition of a mitogen many events are triggered in the lymphocyte and these early events commit the cells to blastogenesis (14). Our kinetic experiments show that the same concentration of exogenous PG which was capable of causing suppression at $0 \mathrm{hr}$ failed to show suppression of mitogenesis when added $16 \mathrm{hr}$ after Con A stimulation. This experiment clearly demonstrates that significant concentrations of PG must be present very early in the course of mitogenesis to be able to influence the process. Therefore, the concentrations of PGs measured at the end of a 48-hr 
culture period may not bear much relevance to the events taking place early in mitogenesis.

Recent evidence indicates that picomole quantities of $\mathrm{PGE}_{2}$ are produced within $1 \mathrm{hr}$ of stimulation with PHA. However, inhibition of this early $\mathrm{PGE}_{2}$ production by IND failed to alter the mitogenic responses of lymphocytes (15), thus supporting our view that the small amount of $\mathrm{PGE}_{2}$ produced during the first few hours of mitogenesis is incapable of influencing the lectin-induced response.

If our proposition that endogenously produced PGs are incapable of influencing the mitogenic process is correct, it follows that the effect of IND observed at submitogenic doses cannot be explained on the basis of PG synthetase inhibition. Is it then possible to explain the effect of IND on some other pharmacological basis? Recently, IND has been shown to be capable of inhibiting cyclic AMP-dependent protein kinases in submicromolar concentrations (16). The authors caution, "In addition to inhibiting prostaglandin synthetase, indomethacin exerts effects on a variety of enzyme systems at micromolar concentrations or greater, properties which should be kept in mind when using this drug to evaluate the role of prostaglandins in biological events." This caveat seems very appropriate to the interpretation of IND effects on in vitro immunological parameters.

The role of cyclic nucleotides in the regulation of lymphocyte proliferation is widely recognized (14); therefore, an agent which can influence cyclic AMPdependent protein kinases can very well be capable of affecting the mitogenic process induced by lectins. Further, such an action might be susceptible to the dose of the mitogen used. Thus, higher concentrations of mitogen might be capable of obviating the activity of IND on cyclic AMP-dependent protein kinases. Present evidence is insufficient to support a role for cyclic AMP-dependent protein kinases in the mitogenic process. However, in view of the potent action of IND on these proteins, further studies are clearly indicated before we can attribute the effect of IND on mitogenic responses solely to the inhibition of prostaglandin synthesis.

In conclusion, our observations concur with other studies $(6,7)$ that PGs suppress the mitogenic responses of PBL when added to an in vitro culture; however, the use of IND to demonstrate the exclusive effect of endogenously produced PGs has certain pitfalls. There is no convincing evidence at the present time that endogenously produced PGs can influence the mitogenic process in the in vitro culture system. It is our contention that the effects seen on the addition of PGs to in vitro cultures may represent a pharmacological effect and may not have much physiological significance. The inconsistent results of the effect of IND on the mitogenic response reported in the literature may be due to the arbitrary use of several different single concentrations of IND in these experiments. Performing dose-response curves to mitogen with and without IND and comparing the maximal mitogenic responses yields a more consistent result in a given population group, and appears essential when comparing different population groups such as healthy and diseased populations. Furthermore, additional studies on the specificity of the effect of IND are clearly needed before meaningful conclusions can be drawn with regard to the effects of PGs in human physiology and pathology.

Note added in proof: Recently Klimpel et al. (17) reported that Con A and PHA stimulation of lymphocytes resulted in activation of soluble cyclic AMP-dependent protein kinase. The non-mitogenic agglutinins wheat germ agglutinin and Agaricus bisporus lectin failed to cause such activation. They conclude that the activation of cyclic AMP-dependent protein kinase may be one of the early events 
linked to and regulating lymphocyte mitogenesis. This observation supports our contention that drugs capable of affecting the activity of the cyclic AMP-dependent protein kinase (e.g., IND) can modulate the lectin-induced mitogenic processes and such modulation is likely to be dependent upon the concentration of the mitogen.

\section{ACKNOWLEDGMENTS}

This investigation was supported by American Cancer Society Grant IM-126; Public Health Service Grants AG-00541, CA-19267, and CA-17404; and the Special Projects Committee, Memorial Sloan-Kettering Cancer Center. We wish to thank Dr. William Lands for useful discussions during the preparation of this manuscript.

\section{REFERENCES}

1. Melmon, K. L., Weinstein, Y., Poon, T. C., Bourne, H. R., and Insel, P. A., In "Immunopharmacology, Comprehensive Immunology" (J. W. Hadden, R. G. Coffey, and F. Spreafico, Eds.), Vol. 3, pp. 331-356. Plenum, New York, 1977.

2. Pleus, I.. M., and Strausser, H. R., Iife Sci. 20, $903,1977$.

3. Webb, D. R., and Nowowiejski, I., Cell Immunol. 33, 1, 1977.

4. Lichtenstein, L. M., Gillespie, E., Bourne, H. R., and Henney, C. S., Prostaglandins 2, 519, 1972.

5. Bray, M. A., Gordon, D., and Mosley, J., Brit. J. Pharmacol. 52, 453P, 1974.

6. Goodwin, J. S., Bankhurst, A. D., and Messner, R. P., J. Exp. Med. 146, 1719, 1977.

7. Goodwin, J. S., Messner, R. P., Bankhurst, A. D., Peake, G. T., Saiki, J. H., and Williams, R. C., N. Engl. J. Med. 297, 963, 1977.

8. Goodwin, J. S., and Messner, R. P., Clin. Res. (Abstract) 26(2), 121A, 1978.

9. Novogrodsky, A., Rubin, A. L., and Stenzel, K. H., J. Immunol. 122, 1, 1979.

10. Böyum, A., Scand. J. Clin. Lab. Invest. 21 (Suppl. 97), 77, 1968.

11. Goodwin, J. S., Messner, R. P., and Peake, G. T., J. Clin. Invest. 62, 753, 1978.

12. Cunningham-Rundles, S., Hansen, J. A., and Dupont, B., In "Clinical Immunobiology" (F. H. Bach and R. A. Good, Eds.), Vol. 3, pp. 151-194. Academic Press, New York, 1976.

13. Ferraris, V. A., and DeRubertis, R. F., J. Clin. Invest. 54, 378, 1974.

14. Hadden, J. W., In "Immunopharmacology" (J. W. Hadden, R. G. Coffey, and F. Spreafico, Eds.), pp. 1-28. Plenum, New York/London, 1977.

15. Phillips, C. A., Girit, E. Z., and Kay, J. E., FEBS Letters 94, 115, 1978.

16. Cantor, H. S., and Hampton, M., Nature (London) 276, 841, 1978.

17. Klimpel, G. R., Byus, C. V., Russell, D. H., and Lucas, D. O. J. Immunol. 123, 817, 1979. 